\title{
Homotopy type of the complement of an immersion and classification of embeddings of tori
}

\author{
M. Cencelj, D. Repovš and M. Skopenkov*
}

This paper is devoted to the classification of embeddings of higher dimensional manifolds. This subject was actively studied in the sixties [1], [2] and there has been a renewed interest in it in recent years [3] - [5]. Investigation of this problem started with consideration of knots $S^{q} \rightarrow S^{m}$, for which an explicit classification in some dimensions, and a complete rational classification in codimension $\geq 3$ was obtained:

Theorem 1. [1] Assume that $q+2<m<\frac{3}{2} q+2$. Then up to isotopy the set of smooth embeddings $S^{q} \rightarrow S^{m}$ is infinite if and only if $q+1$ is divisible by 4 .

We study the case of embeddings $S^{p} \times S^{q} \rightarrow S^{m}$. We call such embeddings knotted tori. Classical special case of knotted tori are links (see Figure 1a). The investigation of knotted tori is a natural next step after knots and links because of the handle decomposition of an arbitrary manifold.

The set of knotted tori in the the space of sufficiently high dimension, namely in the metastable range $m \geq p+3 q / 2+2, p \leq q$, was explicitely described in [4]. The metastable range is a natural limit for the classical methods of embedding theory. The aim of this note is to present an approach which allows for results in lower dimension:

Theorem 2. Assume that $p+4 q / 3+2<m<p+3 q / 2+2$ and $m>2 p+q+2$. Then the set of smooth embeddings $S^{p} \times S^{q} \rightarrow S^{m}$ up to smooth isotopy is infinite if and only if either $q+1$ or $p+q+1$ is divisible by 4 .

Our approach to classification is based on investigation of immersions and the homotopy type of their complements.

Let us give some necessary definitions. Fix points $u \in S^{p}$ and $v \in S^{q}$ and a ball $B=B^{p+q} \subset S^{p} \times S^{q}$ which does not intersect the meridian $S^{p} \times v$ and the parallel $u \times S^{q}$ of the torus (see Figure 1b). A piecewise smooth map $F: S^{p} \times S^{q} \rightarrow S^{m}$ is called an almost embedding, if $F$ is a smooth embedding outside the ball $B$, and $F B \cap F\left(S^{p} \times S^{q} \backslash B\right)=\varnothing$. An almost isotopy is defined analogously, only $B$ is replaced by $B \times I$. Denote by $\mathcal{M}$ the set of all almost embeddings $F: S^{p} \times S^{q} \rightarrow S^{m}$ up to almost isotopy. As it was shown in [5] the set $\mathcal{M}$ has a natural group structure.

The description of the group $\mathcal{M}$ is a much simpler problem than the classification of embeddings and it is done by classical methods. Thus Theorem 2 is reduced to the following problem: to determine, when a given almost embedding

\footnotetext{
* Cencelj and Repovš were supported in part by the Slovenian Research Agency Program No. 101-509. Skopenkov was supported in part by President of the Russian Federation grant NSh-4578.2006.1, Agency for Education and Science grant RNP-2.1.1.7988, Russian Foundation for Basic Research grants No. 02-01-00014, 05-01-00993, 06-01-72551, 07-01-00648, and INTAS grant No. 06-1000014-6277.
} 
is almost isotopic to an embedding. Our main lemma asserts that the complete obstruction to this lies in the finite group:

Lemma 3. Let $p+4 q / 3+2<m<p+3 q / 2$ and $m>2 p+q+2$. Then there is a homomorphism $\beta: \mathcal{M} \rightarrow \mathcal{G}$ into a finite group $\mathcal{G}$, with the following property: if $\beta(F)=0$ then $F$ is almost isotopic to an embedding (smooth outside B).

We give a sketch the proof of Theorem 2 in the case when $q+1$ is divisible by 4 under assumption that Lemma 3 has been proved. It can be shown that the group $\mathcal{G}$ is infinite in this case (and finitely generated). Then the group $|\mathcal{G}| \cdot \mathcal{M}$ is also infinite. For each $F \in|\mathcal{G}| \cdot \mathcal{M}$ we have $\beta(F)=0$, thus each such $F$ is almost isotopic to an embedding (smooth outside the ball $B$ ). By smoothing these embeddings we get infinitely many distinct smooth embeddings $S^{p} \times S^{q} \rightarrow S^{m}$.

Proof of Lemma 3 is based on the following 3-step construction (see Figure 2):

Step 1. Construction of a web $D^{p+1}$. Let $F: S^{p} \times S^{q} \rightarrow S^{m}$ be an almost embedding. Glue a disc $D^{p+1} \subset S^{m}$ to the meridian $F\left(S^{p} \times v\right)$, whose interior is missing the rest of the torus $F\left(S^{p} \times S^{q}\right)$. This is possible by general position, because $m>2 p+q+2$. We call such a disc $D^{p+1}$ is a web.

Step 2. Construction of a web $D^{q+1}$. Using general position we cannot construct the second web $D^{q+1} \subset S^{m}$ glued along the parallel $F\left(u \times S^{q}\right)$. So let us assume initially that such a web exists.

Step 3. Conical Construction. Remove from the sphere $S^{m}$ a neighborhood of the union of the webs. By general position generically the webs have a unique common point, therefore as a result we obtain an $m$-dimensional ball $D^{m}$. We may assume that $D^{m} \cap F\left(S^{p} \times S^{q}\right)=F B$. By replacing $F B$ by the cone over $F \partial B$ inside $D^{m}$, we transform $F$ to the required embedding.

The outline of the rest of the proof of Lemma 3 is as follows. The desired homomorphism $\beta: \mathcal{M} \rightarrow \mathcal{G}$ is the obstruction to existence of the web $D^{q+1} \subset$ $S^{m}$. This obstruction lies in the group $\pi_{q}\left(D^{m}-\operatorname{Im} F, \partial D^{m}-\operatorname{Im} F\right)$, where $D^{m}$ is the complement of a neighborhood of the web $D^{p+1}$. This group is evaluated by the methods of $[3 ; \S 4]$, and in our case it is finite.

[1] N. Habegger and U. Kaiser, Topology 37:1 (1998), p. 75-94.

[2] A. Haefliger, Ann. Math. 83:3 (1966), p. 402-436.

[3] A. Haefliger, Comment. Math. Helv. 41 (1966-67), p. 51-72.

[4] A. Skopenkov, Comment. Math. Helv. 77 (2002), p. 78-124.

[5] A. Skopenkov, Classification of embeddings below the metastable dimension, preprint, http://arxiv.org/ math.GT/0607422 

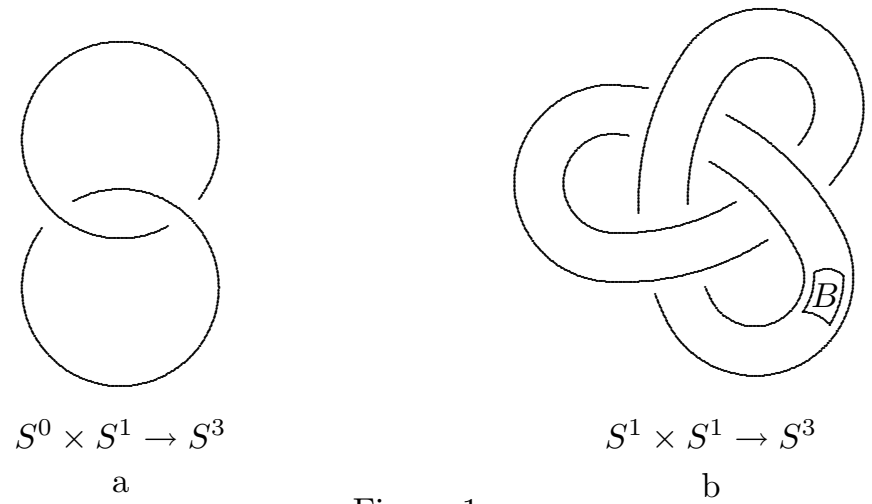

Figure 1 


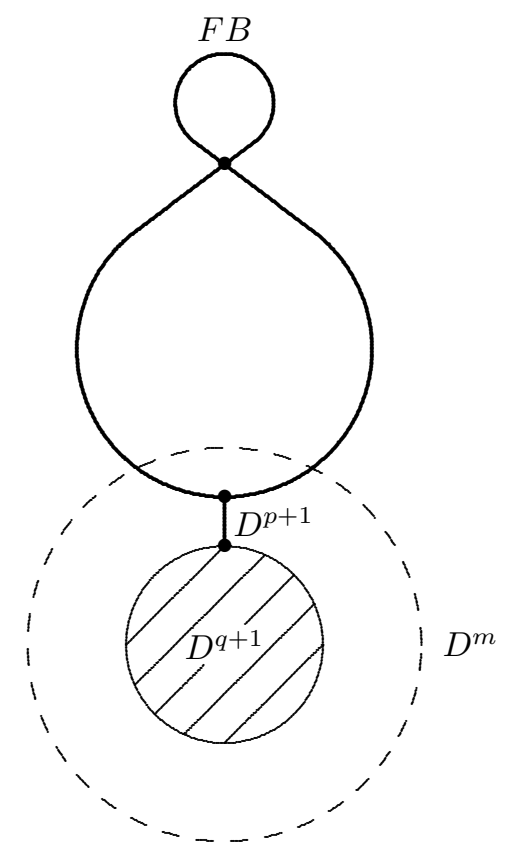

Figure 2 